\title{
Supporting Users in Creating Pedagogically Sound Personalised Learning Objects
}

\author{
Aoife Brady, Owen Conlan, Vincent Wade and Declan Dagger \\ School of Computer Science and Statistics, \\ Trinity College, Dublin, Ireland \\ \{Aoife.Brady, Owen.Conlan, Vincent.Wade, Declan.Dagger\}@cs.tcd.ie
}

\begin{abstract}
Successful eLearning is predicated on the application of pedagogies appropriate to online education that respond to the capabilities and needs of the learners. Typically, designing and assembling personalized Learning Objects that respond to the pedagogical needs of a variety of different learners is an expensive and time-consuming process requiring both domain and educational expertise. Educators have the domain expertise and formal or informal pedagogical knowledge to create quality Learning Objects. However, they lack the tools and often the specific knowledge of online pedagogical approaches that make it time efficient for them to do so. This paper describes the motivation behind, the workflow supported by and the evaluation of the LO Generator, a tool that offers personalized support and scaffolding for users, who are not necessarily content creation or pedagogical experts, in assembling pedagogically sound personalized Learning Objects.
\end{abstract}

Keywords: Personalization, Pedagogy, eLearning, Learning Object Creation

\section{Introduction}

One of the key difficulties in achieving a large-scale take up of Adaptive Hypermedia for eLearning is the cost, complexity and technical barriers to allow non technical teachers and learners to design their own adaptive eLearning experiences. The principle of 'one size not fitting all' is evident not only in static learning content, but also in adaptive content, such as Adaptive Hypermedia, that is created within a particular context that may not suit other uses. Over the last few years a number of adaptive eLearning tools for authoring have begun to appear as witnessed by the success of the $\mathrm{A} 3 \mathrm{H}$ Workshop [1]. A number of these tools have been little more than technical editors to allow for the generation of adaptive content [2]. Others have focused on designing complete adaptive courses, e.g. ACCT [3], WHURLE [4]. However, in blended learning a frequent requirement from teachers, academics and end users [5] is for a specific learning resource on a particular topic that is capable of adapting to the pedagogical needs of individual students. This requirement for finer grained learning objects should be satisfied with minimal user design effort. That said, this effort needs to be balanced against a requirement for pedagogically appropriate and adaptive learning objects (LO) that meet the needs of the end user. This paper describes the LO Generator, a tool that provides personalized support to users in the creation of pedagogically sound personalized Learning Objects. 
This research is focused on developing a unique pedagogical tool to allow end users to generate personalized learning objects on the fly. These learning objects will be semi-automatically generated for a user (either a learner or a teacher) with direct pedagogical and design guidance offered. This process is both empowering and effective as the user can create a learning object that is not only specific to their needs as an educator or learner, but also personalized to learning preferences. This personalization will take two different forms; the personalization of the process of the generation of the learning object, thus tailoring the options that the LO composer is offered, and also the ability to add personalization to the learning object that is generated. The need for a system of this type is driven by the general situation that when you, as a user, search for and retrieve a piece of content the likelihood is that you will not get back exactly what you require. There is a need to bridge this gap between what is requested, what is returned and what the user actually wanted. The aim of this research is to provide pedagogically sound, personalized and context sensitive learning object composition on the fly, enabling both teachers and learners to manipulate learning objects suggested by the system to produce exactly what they require.

The research in this paper extends existing adaptive composition tool research by addressing the problems of personalizing the design process, enabling end user personalization design and more rigorously integrating pedagogical strategies and techniques into adaptive eLearning composition.

This paper describes the pedagogically sound and user-friendly workflow supported by the LO Generator. The need for this type of workflow is placed within the context of the state of the art in this area. The paper then describes how personalization is applied and presents the evaluation and its results. The paper concludes by describing the future work that will be carried out on this system.

\section{State of the Art}

This section briefly reviews and compares four recent authoring systems aimed at producing eLearning courses. Each occupies a subtly different niche in the area of Adaptive Hypermedia Authoring Systems. My Online Teacher (MOT) [6] is a tool developed at Eindhoven University of Technology for authoring Adaptive Hypermedia courses. MOT utilizes a three-layer model for authoring adaptation [7] that provide a conceptual hierarchical layer of atomic and composite concepts, a lesson layer, which provides the manner and sequencing of the concepts, and a third layer which consists of an adaptation engine. Its output is at the course level and matches the AHA! Model (AHAM) closely. A consequence of this is that the tool does not explicitly support specific pedagogies and requires the author to have some knowledge of the AHA! system upon which the courses are deployed.

The Dialog Plus learning design toolkit [8] approaches authoring from a very different perspective. It is a toolkit that guides users through the process of designing pedagogically sound learning activities, known as learning nuggets. These nuggets are made up of various tasks that are to be undertaken in a specified context in order to 
attain certain learning outcomes [9]. The toolkit is highly pedagogic as it offers various pedagogical approaches. After the user has selected an approach, the toolkit provides guidance to the user in building the individual nuggets. Unlike MOT, Dialog Plus does not have a specific target platform on which it is deployed and as a consequence aids the user in assembling the model of a course rather than in deploying a specific course instance.

The ASK Learning Designer Toolkit (ASK-LDT) [10] provides a graphical authoring system to create learning scenarios based on IMS LD Level B [11]. This tool is much more programmatic in nature than the previous tools and requires the author to have a strong knowledge of the IMS LD specification and its capabilities. As such there is no explicit pedagogic guidance offered to the author, but it could be argued that IMS LD has in built pedagogical biases. The authoring process supported in ASK-LDT consists of several steps [2]. The first allows the definition of the pedagogical elements and is followed by the definition of the environment. The next step is the design of the learning scenario, after which comes the statistical analysis and is finished by the content packaging step. ASK-LDT is highly focused on producing IMS LD compliant outputs and as such has a number of platforms (e.g. Reload [12]) that are capable of playing the resulting LDs.

The final authoring system discussed in this section is the Adaptive Course Construction Toolkit (ACCT) [3] is a system that allows a course developer to create both adaptive and non-adaptive activity-oriented courses based on sound pedagogical strategies. The ACCT offers many different tools available to the course developer: a concept space/domain ontology builder, a custom narrative builder, a content package assembler, learning resource repository interactivity and also a real time course test and evaluation environment. ACCT has two key features; it offers an abstracted pedagogy-based framework in which to construct courses and it enables the courses to be deployed to APeLS [13], as well as in IMS LD format. Its support of IMS LD is not as rich as that offered in ASK-LDT, nor is the pedagogic support offered by its framework as detailed as that in Dialog Plus, however ACCT strikes a balance that enables pedagogically sound courses to be created and deployed.

Table 1. Comparing MOT, Dialog Plus, ASK-LDT and ACCT

\begin{tabular}{|c|c|c|c|c|}
\hline Feature / System & MOT & ACCT & Dialog Plus & ASK-LDT \\
\hline Learner as Designer & No & No & No & No \\
\hline Teacher as Designer & $\begin{array}{l}\text { Yes (Knowledge of } \\
\text { AHAM required) }\end{array}$ & Yes & Yes & $\begin{array}{l}\text { No (Knowledge } \\
\text { of LD Required) }\end{array}$ \\
\hline $\begin{array}{r}\text { Produce Individual } \\
\text { LOs }\end{array}$ & No & No & No & No \\
\hline Produce Courses & Yes (in AHA!) & $\begin{array}{c}\text { Yes (in APeLS } \\
\text { or LD) }\end{array}$ & $\begin{array}{c}\text { Yes (as } \\
\text { Activities) }\end{array}$ & Yes (as LD Act) \\
\hline $\begin{array}{r}\text { Explicit Pedagogic } \\
\text { Guidance }\end{array}$ & No & Yes & Yes & No \\
\hline 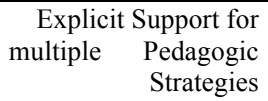 & No & Potentially & Potentially & No \\
\hline
\end{tabular}


The above table summarizes the capabilities of each of the four authoring systems mentioned. As may be seen from this table each system occupies a different niche and offer authors different features. It is worth noting that none of the systems offer support for creating learning objects.

As a final piece of related work Generative Learning Objects (GLOs) [14] are based on the idea that for LOs to be adaptable, the structure of the learning design needs to be separated from the content. The construction of a GLO is broken up into two parts. The first part is the creation of a Learning Object Template. This template encompasses the learning design and is created by a team of experts, students, an artist and a facilitator. The job of the facilitator is to ensure that the design produced is suitable for a GLO. Once the Template is created web based forms allow either a tutor or a student to instantiate the GLO by adding subject specific content to the Template. This work is referenced here as the LO Generator presented in this paper is also template-based, but takes a fundamentally different approach.

\section{Workflow of the LO Generation Process}

As may be seen from the State of the Art section there is an unexplored niche in the area of personalized LO creation. However, the most successful systems not only present a tool that enables the creation of a personalized offering, but do so in a pedagogically supportive manner that scaffolds the non-expert. Supporting non-expert users in creating learning objects for their own or others consumption requires a logical and easy to follow workflow to be implemented. This section describes the workflow for creating pedagogically sound personalized learning objects that is enabled in the first iteration of the LO Generator. A wizard was implemented to allow the user to create a Learning Object by following a set of logical steps. The first two steps involve the user interacting with two separate ontologies, a learning domain concept ontology and a learning outcomes ontology, in order to refine the scope of the Learning Object to be created.

Step 1 allows the user to select the learning domain concepts that they would like covered by their LO. In this step the user is presented with a list of high level domain concepts, for example, The Structure of the Human Eye and How We See, in the domain of Human Vision. After selecting a high level concept, this concept is then decomposed by the system with the help of a domain specific concepts ontology. This decomposition allows the high level concept to be broken down into lower level concepts allowing the user to specify exactly the low level concepts that they require. For example, when The Structure of the Human Eye is selected as a high level concept, it may be decomposed into several low level concepts, such as cornea, aqueous humour, iris, etc. The user can then select or deselect the various concepts that they would like included.

After the user has specified the domain concepts that they wish to be included in the LO, they are invited to move on to the next step. During this step, the user is asked to choose the overall learning outcome of the LO. These overall learning outcomes are based on Bloom's Taxonomy [15] which comprises Knowledge, 
Comprehension, Application, Analysis, Synthesis and Evaluation. When a user selects one of these high level outcomes, in a similar manner to the high level domain concept in the previous step, the outcome may then be decomposed into various learning events or activities which support a pedagogically sound approach to fulfilling these outcomes. This decomposition is performed using the learning outcomes ontology. The user can again select or deselect any of these learning events or activities as they want. Each learning activity and event is accompanied with supporting text that helps guide the user towards pedagogically appropriate ones for their outcomes. This screen is shown in Figure 1, where the learning outcome is chosen on the left and the specific activities are chosen on the right. These activities can be turned on and off for each selected sub-concept from Step 1. In this example the higher level, Knowledge, outcome is selected and this is broken down into the activities introduce, explain and self test.

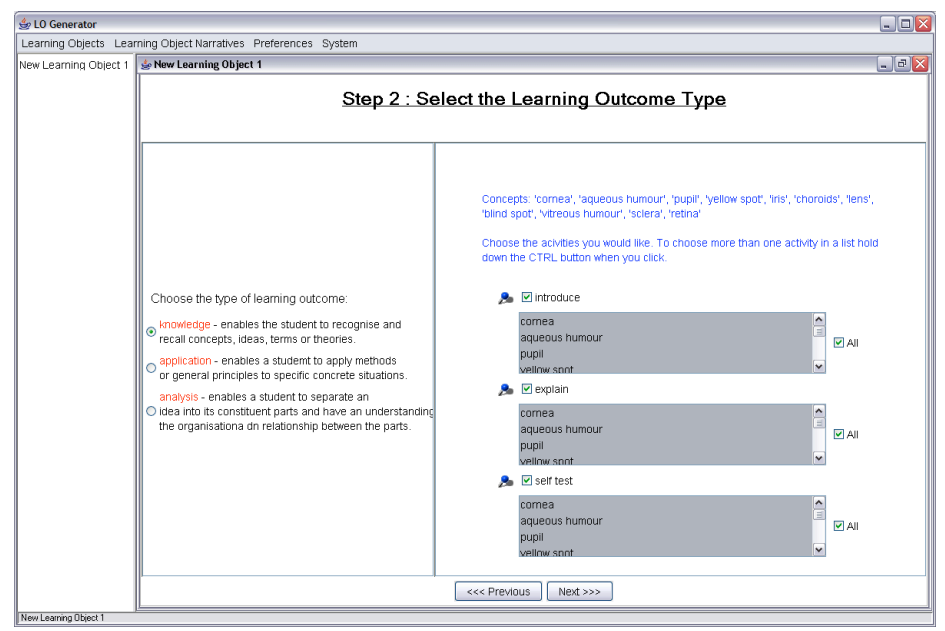

Fig. 1. Specifying the Activities

When these two steps are completed and the user is satisfied with their choices, the LO Generator then calls APeLS [13] to search for all Learning Object Narratives that fulfils the chosen learning outcome(s) across the desired concepts and utilises the learning events and activities specified by the user. A LO Narrative is a structure which comprises a pedagogical strategy and is composed of a set of rules which governs the selection and sequencing of learning activities and content. When these LO Narratives are chosen, they are presented to the user (see Figure 2) for inspection and selection. The LO Narrative that the system deems to be most relevant is highlighted, although the user can change the selected one if they feel that another matches their requirements more closely. The user can access information about each of these LO Narratives, such as the pedagogical description, the author, etc., as well as compare the narratives so as to make an informed decision. The one chosen in our example is the one that encompasses the three required learning activities chosen above 


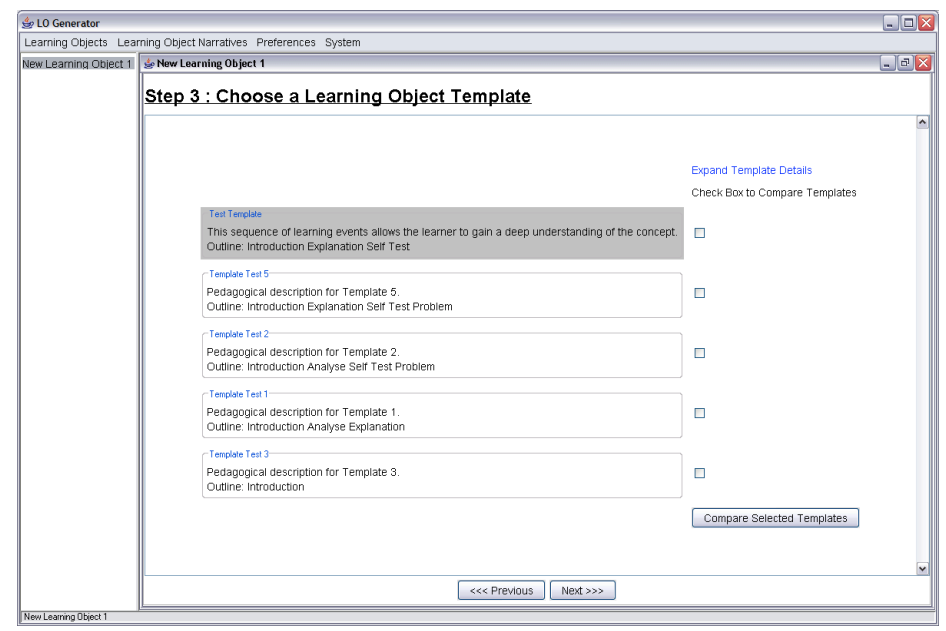

Fig. 2. Choosing the LO Narrative

When the LO Narrative is chosen by the user, the system moves onto the next step. At this stage a skeleton LO is created with actual pre-selected content assets that would be used in the generated LO. These content assets are chosen based on the pairings of each low level concept, a complementary learning event or activity. The user can make changes to this LO skeleton by reordering or deleting these pairings. This stage is shown in Figure 3, with the tree on the left representing the skeletal structure of the LO. Using drag and drop the activities can be re-sequenced in any order desired. At any stage during the generation process the user can choose to go backwards and change any of their selections.

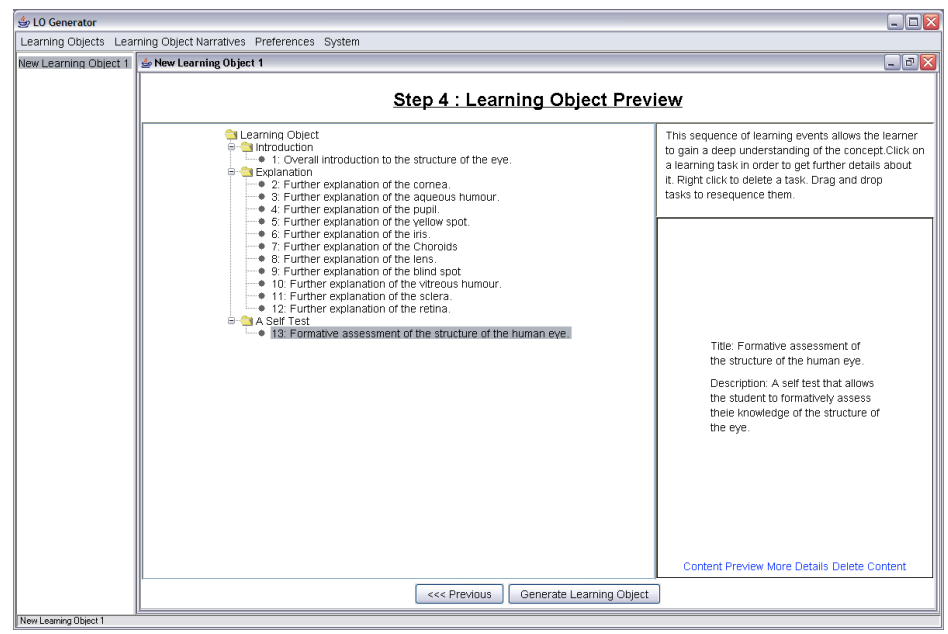

Fig. 3. Confirming the structure of the LO 
The support for the two forms of adaptivity offered by the LO Generator are described in the next section, but it is at this stage that the second, personalization of the LO, will be applied.

Once the user is satisfied with the skeleton that is presented, they can then choose to generate the learning object. When the LO is generated it is output as an IMS Learning Design (IMS LD) [11] compliant manifest using an XSLT transform. An IMS Content Package [16] is created that combines this manifest with the appropriate learning assets and it is this package that the user is offered as a download at the end of the generation process. This package can then be they played in an IMS LD compliant player such as Reload [12].

\section{Supporting Personalization in LO authoring}

Personalization in this workflow is represented in two different ways. Firstly, there is the personalization of the experience of the LO composer. Through repeated use of the LO Generator the desires of the composer will be observed and automatically accounted for. These desires are manifest as frequent deviations from the recommendations offered by the LO Generator. For example, if the composer frequently adds specific learning activities to all suggested structures this pattern will be recorded and in future these additions would be automatically performed. The second form of personalization is within the LOs produced by the system. When the composer is creating an LO they have access to several adaptive LO (sub-) Narratives that they may compose together to provide adaptivity, e.g. to prior knowledge, across the learning activities added to the LO Generator.

In order to support the two layers of personalization offered by the system the authoring environment given by the LO Generator is designed to be responsive to the authors needs and desires. The first form of personalization, i.e. adapting to their authoring style and needs, is performed by assessing their pattern of LO creation. This pattern may only be observed through repeated use of the tool and is manifest as recording the modifications the author makes to the templates that are suggested to them. When the conditions are met for a similar template to be given these differences are analysed for commonalities. The most popular commonalities are added to the template automatically and are tagged as user preferred modifications.

To facilitate the second layer of personalization, the tool itself will provide functionality to the user to add personalization to the LO presented in the final step, before generation. The user will be able to select the concept and activity that they would like to enable adaptivity on and will be able to choose the type of adaptivity they would like, for example, adaptivity due to prior knowledge or progressive adaptivity. This personalization will be available at two levels, the first being to add the adaptivity to the entire LO and the second being the ability to add the adaptivity to elements within the LO. This personalization is facilitated by the LO Narratives which will be designed to encompass rules and conditions to ensure this adaptivity is appropriate. The LO Narratives, as the name suggests, are derived from narratives in the multi-model metadata driven approach [17], which enable a hierarchical layering 
of narratives and sub-narratives to facilitate personalization. Concept domain and adaptation are reconciled through LO Narratives applied at design time by the LO Generator and are added to the LO generated. This approach facilitates scalable and generic adaptivity, but has the potential downside of enabling inappropriate personalizations. This is why the on the fly preview capability and pedagogical scaffolding are important to ensure the generated LO is suitable for the author's needs.

\section{Trial and Evaluation}

This section provides an overview of the trial and evaluation process carried out in order to assess the suitability of the LO Generator. The initial results are overwhelmingly positive, particularly in the area of flexibility of the tool and the suitability of the produced LOs, where the trial participants expressed satisfaction with both the process and product. Many of the advanced features of personalisation were not featured at this stage, but the fundamental pedagogical process and learning object construction paradigms were in place.

An initial experiment for the LO Generator took place in early August 2007 with the aim to trial the prototype LO Generator with five users to get their feedback on the usability of the software in relation to the user interface and importantly on the perceived educational effectiveness of the tool. The users that participated in the evaluation all had teaching experience. This tool has been designed to be used by both teachers and learners, but it was decided to initially trial it with teachers. During the trial the users were asked to complete two surveys. The first dealt primarily with usability and user interface issues and will only be briefly discussed. The more pertinent survey with regard to the focus of this paper addressed the perceived educational benefit of LO Generator.

Assessing the fundamental usability of the LO Generator as a tool is important in order to ensure its basic technical suitability for the purpose of constructing personalized learning objects. The usability evaluation questionnaire was originally developed in Trinity College, Dublin as part of an approach to evaluate educational courseware [18]. During this survey the users were asked to agree or disagree with statements about the user interface in the areas of naturalness, navigation, user support, consistency, non redundancy and flexibility. The general responses given were very positive with all users agreeing that the LO Generator was usable for its envisaged purpose. The primary comments centered around the quantity and quality of instructions and scaffolding available in the use of the tool. Most users agreed that they were sufficient, but a minority desired more.

The second survey in the evaluation concentrated on the perceived educational effectiveness of the system and the composed learning object. The goals of this evaluation were to determine if the Learning Object composed by the system reflected what the user wanted (Goal 1), to discover whether the composed Learning Object is usable (Goal 2) and to discover whether the teacher felt that they had control over the composition and whether this was the composition that they wanted, i.e. could they produce a Learning Object for their needs (Goal 3). 
Each of these three goals was broken down into a series of discrete objectives upon which the users would be questioned. The results of this survey were mixed but overall positive about the use of the LO Generator.

With reference to the first goal, the users responded that their initial educational requirements were fulfilled while using the LO Generator. They believed that all the topics they selected were covered in the resulting LO and for the most part were taught in the manner expected. Unfortunately, at this stage the manner they were taught in was linear which was pointed out as being a drawback. The sequence of activities matched their expectations and they felt that they could specify the required topics though they were limited by the selection of topics available. On a negative note it was commented that the choice of activities did not necessarily fulfil the requirements of the learning outcome, but maybe would suffice when the system was complete and more content available for later trials. It is planned that further implementations of the tool will provide more available activities.

Regarding the second goal, which was to determine the usability of the composed Learning Object, for the most part the users thought that they had sufficient information to make use of the LO. However, a minority believed that there was not enough information to make use of the LO. They were then asked whether the design process resembled one that they would normally use to design a LO. Those who had actually given some thought to designing an LO before answered yes. They reported that the steps involved in the process were appropriate and the flow between them was logical. They reported that the mapping between concepts and content was readily understood, but that the mapping between the learning outcomes and the activities was not necessarily as transparent. This mapping may require some scrutable reasoning to explain to the user why these activities were chosen. When asked whether they would have the confidence to actually use the LO as part of their course they all answered yes, but with some stipulations. One mentioned that it would be useful to have proper training with using the tool, another mentioned that she would need to see the end result before committing and to have relevant course material available.

With respect to the third goal, the users were first asked whether they could compose the Learning Object that they wanted using this tool and they all answered yes. Using the LO Generator to generate an LO was not thought to be a difficult process. They felt that they had adequate control over the various stages, except for the sequencing of activities which got a mixed response, mainly due to the usability of the sequencing tool. They agreed that the granularity of both topics and activities were suitable. They liked the fact that they could define the activities they wanted, but would like a way of adding their own as they believed that there were not enough available. To qualify this it was expressed that this could be remedied with more development. With regard to the learning objectives, there was a mixed response as to whether you could specify the ones that you wanted. It was reported that it would be great to be able to specify clearer objectives and also it would be good to be able to specify or add your own. It was also reported that the users would like to be able to create their own LO Narratives.

In summary the reaction to the LO Generator as a tool was a positive one. As regards its usefulness the users believed that they could see the potential in a system 
like this but would like some additional features to be added to make it more useful. The addition of a way to personalise the finished LO would also be welcomed.

\section{Conclusions}

This paper has detailed the workflow of the current version of the LO Generator which has focused on the importance of appropriate pedagogical scaffolding and usability. The LO Generator empowers an author who is not a pedagogical expert in assembling a Learning Object on the fly to suit their needs. The next version is under development with the addition of the personalization components being the main focus. With this personalization in place, the vision of the generation of fully adaptive learning objects created in an adaptive environment will become a reality. Plans for a second phase of evaluation are in place to discover the usability and educational effectiveness of this completed approach.

\section{References}

1. A3H International Workshop on Authoring of Adaptive and Adaptable Hypermedia. http://www.win.tue.nl/ acristea/A3H/

2. Karampiperis, P., Sampson, D.G.: A Flexible Authoring Tool Supporting Learning Activities. IADIS International Conference Proceedings of the IADIS International Conference on Cognition and Exploratory Learning in Digital Age, CELDA 2004, pp. 5158. Lisbon, Portugal, 2004

3. Dagger, D., Wade, V., Conlan, O.: Personalisation for All: Making Adaptive Course Composition Easy. Educational Technology \& Society, Vol. 8, No. 3, pp. 9-25, 2005.

4. Web-based Hierarchical Universal Reactive Learning Environment (WHURLE). http://whurle.sourceforge.net/

5. Intelligent Distributed Cognitive-based Open Learning System for Schools (iClass), European Commission FP6 IST Project. http://www.iclass.info.

6. Cristea, A.I., De Mooij, A.: Adaptive Course Authoring: My Online Teacher. $10^{\text {th }}$ International Conference on Telecommunications, ICT '03, pp. 1762- 1769. Papeete, French Polynesia, IEEE, 2003.

7. Cristea, A., Aroyo, L.: Adaptive Authoring of Adaptive Educational Hypermedia. 2nd International Conference on Adaptive Hypermedia and Adaptive Web-Based Systems, AH 2002, pp. 122 - 132, Malaga, Spain, Springer-Verlag, 2002.

8. Conole, G., Fill, K.: A learning design toolkit to create pedagogically effective learning activities. Journal of Interactive Media in Education, 2005.

9. Bailey, C., Zalfan, M.T., Davis, H.C., Fill, K., Conole, G.: Panning for Gold: Designing Pedagogically-inspired Learning Nuggets. Educational Technology \& Society, Vol. 9, No. 1, pp. 113-122, 2005

10. Sampson, D.G., Karampiperis, P., Zervas, P.: ASK-LDT: A Web-Based Learning Scenarios Authoring Environment Based on IMS Learning Design. International Journal on Advanced Technology for Learning (ATL)), pp. 207-215, ACTA Press, October 2005.

11. IMS Global Learning Consortium Learning Design Specification (IMS LD). http://www.imsglobal.org/learningdesign/.

12. Reusable eLearning Object Authoring and Delivery (RELOAD). http://www.reload.ac.uk/ 
13. Conlan, O., Wade, V. Evaluating the Multi-model, Metadata-driven Approach to producing Adaptive eLearning Services. Third International Conference on Adaptive Hypermedia and Adaptive Web-Based Systems (AH2004) Proceedings, Eindhoven, The Netherlands (2004)

14. Morales, R., Leeder, D. \& Boyle, T.: A Case in the Design of Generative Learning Objects (GLOs): Applied Statistical Methods. In P. Kommers \& G. Richards (Eds.), Proceedings of World Conference on Educational Multimedia, Hypermedia and Telecommunications 2005 (pp. 2091-2097). Chesapeake, VA: AACE.

15. Major Categories in the Taxonomy of Educational Objectives (Bloom 1956). http://faculty.washington.edu/krumme/guides/bloom1 html

16. IMS Global Learning Consortium Content Packaging Specification (IMS CP). http://www.imsglobal.org/content/packaging/

17. Conlan, O.; Wade, V.; Bruen, C.; Gargan, M.: Multi-Model, Metadata Driven Approach to Adaptive Hypermedia Services for Personalized eLearning. Second International Conference on Adaptive Hypermedia and Adaptive Web-Based Systems, Malaga, Spain, May 2002

18. Lyng, M.: Experience Improving WWW based Courseware through evaluating User Satisfaction, M.Sc. in Computer Science, Trinity College, Dublin 